\title{
Engagement vs Performance: Using Electronic Portfolios to Predict First Semester Engineering Student Persistence
}

\author{
Everaldo Aguiar, G. Alex Ambrose, Nitesh V. Chawla, Victoria Goodrich, and Jay Brockman \\ University of Notre Dame \\ nchawla@nd.edu
}

\begin{abstract}
As providers of higher education begin to harness the power of big data analytics, one very fitting application for these new techniques is that of predicting student attrition. The ability to pinpoint students who might soon decide to drop out, or who may be following a suboptimal path to success, allows those in charge not only to understand the causes for this undesired outcome, but provides room for the development of early intervention systems. While making such inferences based on academic performance data alone is certainly possible, we claim that in many cases there is no substantial correlation between how well a student performs and his/her decision to withdraw. This is especially true when the overall set of students has a relatively similar academic performance. To address this issue, we derive measurements of engagement from students' electronic portfolios and show how these features can be used effectively to augment the quality of predictions.
\end{abstract}

KEYWORDS: Electronic Portfolios, Student Retention, Early Intervention, Data Fusion, Learning Analytics, Predictive Analytics

\section{INTRODUCTION}

Over the course of many years, the education field has gone through several transformations. As new techniques for both teaching and assessing students emerge, universities and other post-secondary institutions are expected to adapt quickly and begin to follow the new norms. Further, as the needs of our society shift, we often see increased demands for professionals in particular disciplines. Most recently, this phenomenon can be observed with respect to the areas of Science, Technology, Engineering, and Mathematics (STEM).

While creating an environment that stimulates student enrollment in these particular fields is a challenge in itself, preserving high retention rates can be a far more complicated task. As Seidman (2005) highlights, our understanding of retention has considerably changed over time, and efforts to address the issue are ubiquitous in higher education today. Yet, despite the rapid growth of this subject over the last few years, there are clear indications that the complexities involved with helping a highly diverse array of students to succeed are far from being understood.

It is estimated that nearly half of the students who drop out of their respective programs do so within their first year in college (Delen, 2011). Consequently, a clear focus has been directed towards early identification and diagnosis of at-risk students, and a variety of studies using statistical methods, data 
(2014) Engagement vs Performance: Using Electronic Portfolios to Predict First Semester Engineering Student Persistence. Journal of Learning Analytics, 1 (3), 7-33.

mining, and machine learning techniques can be found in recent literature (Thammasiri, Delen, Meesad, \& Kasap, 2013; DeBerard, Spielmans, \& Julka, 2004; Zhang, Anderson, Ohland, \& Thorndyke, 2004; Burtner, 2005; Yu, DiGangi, Jannasch-Pennell, Lo, \& Kaprolet, 2007; Mendez, Buskirk, Lohr, \& Haag, 2008; Li, Swaminathan, \& Tang, 2009; Lin, Imbrie, \& Reid, 2009; Delen, 2010; Zhang, Oussena, Clark, \& Hyensook, 2010).

A downside of these proposed models is that they frequently rely strictly on academic performance, demographic, and financial aid data. There is a wide recognition, however, that the reasons for student dropouts can range based on several other factors outside that scope (Astin \& Astin, 1992; Pascarella, Terenzini, \& Blimling, 1994; Xenos, Pierrakeas, \& Pintelas, 2002; Flowers, 2004; Pascarella, 2006; MacGregor \& Leigh Smith, 2005; Li, Swaminathan, \& Tang, 2009). Moreover, a number of students who are not retained do not exhibit any early signs of academic struggle as per their grades. The inverse is also true, as there are often highly engaged students who despite performing below expectations, remain enrolled. Figure 1 illustrates these two specific groups of students.

In this paper, we focus on remedying the shortcomings that arise when classification models are trained using only student academic performance and demographic data. We collected data that describe the access patterns of first-year engineering students to their personal electronic portfolios, which are dynamic web-based environments where students can list and describe their skills and achievements, and we show how these features correlate to and can help enhance the prediction accuracy of student attrition. In particular, we investigate how measurements of student engagement can be used to decrease miss-prediction rates of instances belonging to the groups highlighted in Figure 1.

The remaining portion of this paper is organized as follows. Section 2 gives an overview of the most recent related literature. Section 3 describes the context in which this study was carried out and gives insight as to our decision to utilize electronic portfolios to measure student engagement. Section 4 expands that idea by highlighting some preliminary observations on the usefulness of ePortfolios. Following, section 5 describes our dataset in detail. The methodology and experimental results are covered in sections 6 and 7 respectively, and a brief discussion of our findings concludes this paper in section 8. 
(2014) Engagement vs Performance: Using Electronic Portfolios to Predict First Semester Engineering Student Persistence. Journal of Learning Analytics, 1 (3), 7-33.

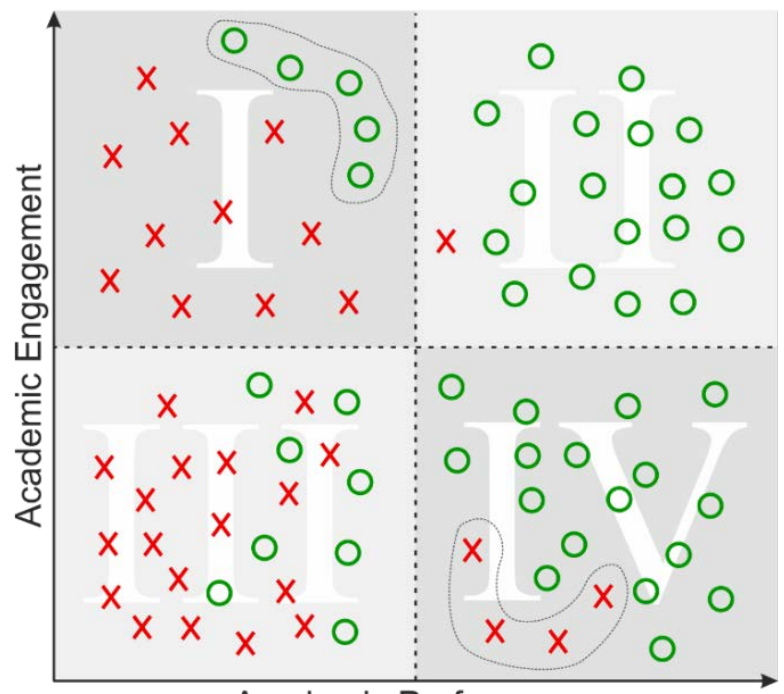

Academic Performance

$X$ Not Retained after First Semester $\bigcirc$ Retained for Second Semester

Figure 1: Low performing/highly engaged students (quadrant I) are often retained. High performing/disengaged students (quadrant IV) may not persist.

\section{RELATED WORK}

From a sociological standpoint, student attrition has been studied in great detail. Seidman (2005) and Tinto (2012) provide comprehensive studies that investigate the causes and consequences of this issue. Though related, this ramification of the literature is outside the scope of this paper. Following, we provide a more elaborate description of the most recent works that utilize student data to create and evaluate prediction models for student attrition.

Early work by DeBerard et al. (2004) combined academic performance, demographics and self-reported survey data of students in an attempt to forecast cumulative GPA using linear regression, and retention rates via logistic equations. The former achieved commendable results while the outcomes of the later were not statistically significant. Contemporary to that, a study by Zhang et al. (2004) showed that high school GPA and math SAT ${ }^{1}$ scores were positively correlated to graduation rates of engineering students, while verbal SAT scores correlated negatively with odds of graduation. Similar findings are reported by Mendez et al. (2008).

A key premise of our work is highlighted by Burtner (2005). After monitoring a group of incoming engineering students over a three year period, the author concludes that while a predictive model based on cognitive variables such as the students' math and science ability can perform relatively well, it would

\footnotetext{
${ }^{1}$ A standardized test widely used for college admissions in the United States 
(2014) Engagement vs Performance: Using Electronic Portfolios to Predict First Semester Engineering Student Persistence. Journal of Learning Analytics, 1 (3), 7-33.

greatly benefit if non-cognitive factors developed during the freshman year were to be incorporated. Lin et al. (2009) validate that idea by showing that the accuracy of their classification models improves after the inclusion of non-cognitive features extracted from a survey.

Yu et al. (2007) utilize decision trees to predict student retention, and among other discoveries, the authors report that in their context, student persistence was more closely related to the students' residency status (in/out of state) and current living location (on/off campus) than it was to performance indicators. Likewise, a sensitivity analysis exercise performed on neural networks, decision trees, support vector machine and logistic regression models by Delen $(2010 ; 2011)$ ultimately concluded that several important features utilized to predict student retention were not related to academic performance.

With the intent of developing a long-term intervention system to enhance student retention, Zhang, Oussena, Clark, \& Hyensook, (2010) tested three different classifiers and observed that the best prediction accuracy for student retention was yield by naive Bayes. Alkhasawneh, (2011) utilizes neural networks to predict first year retention and provides an extensive analysis of his models' performance. Finally, we highlight the recent work by Thammasiri et al. (2013), in which the problem of predicting freshmen student attrition is approached from a class imbalance perspective, and the authors show how oversampling methods can enhance prediction accuracy.

\section{CONTEXT}

\subsection{The College of Engineering}

The University of Notre Dame is a medium-sized, Midwestern, private institution with a traditional student composition, i.e., the vast majority of students complete their undergraduate studies in four years and are in the age range of 18 to 22 . The overall student body is 53 percent male and 47 percent female, while the College of Engineering is approximately 75 percent male and 25 percent female. Firstyear students are admitted to the First-Year of Studies program regardless of their intended future major. Students select their major (whether engineering or something else) near the end of their first year when they register for classes for the upcoming fall semester. Beyond admission/selection into the university as a whole, there are no admission or selection criteria for entering any of the disciplines of engineering; rather, it is based on student interest alone.

With few exceptions, first-year students considering an academic pathway within engineering complete a standard first-year curriculum, including the two-semester course sequence of "Introduction to Engineering," taught within the College of Engineering. Each year the course sequence has enrollments of approximately 450 to 550 students. The course has two main objectives: (1) to expose students to the engineering profession and engineering major options, and (2) to demonstrate the processes of planning, modelling, designing, and executing specified project deliverables. The course curriculum uses 
(2014) Engagement vs Performance: Using Electronic Portfolios to Predict First Semester Engineering Student Persistence. Journal of Learning Analytics, 1 (3), 7-33.

a project-based learning approach, with students completing a total of three group projects across the two-semester sequence. Students are required to attend large lecture sections, which introduce basic concepts needed to complete the projects, and small group (30 to 35 students) learning centres that focus on hands-on learning. For over a decade, the course sequence has included similar material and project-based course assignments, including homework, quizzes, exams, technical reports, and presentations.

\section{2 ePortfolios for Engagement}

The ePortfolios serve as a creative space and a recording system that utilizes digital technologies to allow learners to collect artifacts and examples of what they know and can do, in multiple media formats, using hypertext to organize and link evidence to appropriate outcomes/skills, goals, or standards (Barrett, 2007). ePortfolios capture and document student learning and engagement through their reflection, rationale building, and/or planning. Chen and Black (2010) found ePortfolios generate shared responsibility and ownership of learning between students and instructors since they can be used inside and outside the classroom. They are also available and can be used on and off campus, in face-to-face and virtual environments, and during and after the student's time in college (as a way of practically demonstrating what ABET (Accreditation Board for Engineering and Technology, 2013) refers to as "life-long learning" achievements. Al-Atabi et al. (2011) found the use of ePortfolios to be valuable as an advising tool, allowing students to track the progress of their learning outcomes, to provide documentary evidence, and to be used when they meet regularly with their academic advisors for feedback. Significantly, the use of ePortfolios generates intentional and active learners since students become self-aware and take ownership of their academic progress.

Higher education institutions such as Bowling Green State University (Knight, Hakel, \& Gromko, 2008), La Guardia Community College (Eynon, 2009), University of Barcelona (Lopez-Fernandez \& RodriguezIllera, 2009), Spelman College (Price, 2006), Clemson (Ring, Weaver, Jones, \& others, 2009), Penn State, and Florida State Universities (Yancey, 2009) have begun to implement ePortfolio initiatives to enhance engagement and measure impact through integrating life-wide academic, personal, and professional contexts. Kahu (2013) points out that because of its complex nature, finding a common definition for student engagement across the several fields that study it is a difficult task. The authors subscribe to Kuh et al.'s (2000) student engagement as a construct that measures the alignment between what effective institutions purposefully do (a range of teaching practices and programmatic interventions) to induce and channel students to desired outcomes, compared with what students actually do with their time and energy towards achieving these educationally purposeful activities (Kuh, Hu, \& Vesper, 2000). More specifically, using Kahu's (2013) behavioural dimension of engagement, we focus on the digital observations, or behavioural measurements, of online time on task, effort, and participation.

The ePortfolio platform of our choice is supported by Digication (Digication e-Portfolios, 2013) and its Assessment Management System (AMS). Their paid subscription account not only offers an ePortfolio 
(2014) Engagement vs Performance: Using Electronic Portfolios to Predict First Semester Engineering Student Persistence. Journal of Learning Analytics, 1 (3), 7-33.

platform but also provides a powerful back-end course, program, institution, or inter-institution AMS. Within individual, and across our partnering institutions, the AMS tracks, compares, and generates reports on student progress and performance by standards, goals, objectives, or assignments.

Realizing the importance of having a deep understanding of how students interact with and make use of their electronic portfolios, we worked alongside Digication to develop an automated pipeline for data collection that has since been deployed. We collect student-level data on a daily basis, and while the work described in this paper made use of aggregated datasets collected at the end of the 2012 fall semester, in future we will be able to scale our models for predicting early signs of risk to much more actionable time stamps.

\section{3 ePortfolio Engagement as an Analytic}

For too long much of the emphasis on improving retention has focused solely on the binary metric of retention (yes/no). By focusing on student engagement rather than just predictive variables, after-thefact outcome of retention, or a subjective measurement of learning, the ePortfolio provides a window into the time, energy level, and commitment exhibited by students throughout the trajectory of a given course. The assessment focus on retention is too late to provide an actionable window and improve learning within a course, especially during the first semester of college.

An ePortfolio engagement analytic has important implications for the emerging field of learning analytics. Johnson et al. (2010) define learning analytics as the interpretation of a wide range of data produced by and gathered on behalf of students in order to assess academic progress, predict future performance, and spot potential issues. The goal of learning analytics is to enable educators to understand and optimize learning via an environment tailored to each student's level of need and ability in close-to-real time. Up until now, most of the data sources have been limited to learners' tacit digital actions inside the learning management systems (i.e., discussion forum posts, downloading content to read, login rates, and duration). The ePortfolio tool and platform offers a more authentic environment that could provide a week-by-week measure to identify if and when students are losing engagement and explore why, where, and what is engaging students as well as how they spend their time and energy outside of the class. Therefore, data mining the ePortfolios could generate more effective learning analytics to improve the understanding of teaching and learning, and to tailor education to individual students more effectively.

\section{4 ePortfolio Use in the First-Year Engineering Course}

In the 2012-2013 academic year, ePortfolio assignments were integrated with the traditional course deliverables as a means to guide student reflection on their education. Eleven ePortfolio updates were assigned throughout the academic year. For the course, all students were required to create an ePortfolio following an instructor-designed template. The ePortfolio template included three main 
(2014) Engagement vs Performance: Using Electronic Portfolios to Predict First Semester Engineering Student Persistence. Journal of Learning Analytics, 1 (3), 7-33.

sections, which were each updated over the course sequence:

1 Engineering Advising: Required reflection on their engineering major choice and their progress towards engineering skill areas. Seven skills areas were defined, each relating to ABET accreditation-required outcomes (a-k).

2 Project Updates: Required updates following the completion of each project. Minimally, students were asked to include a picture of their project and a reflection on skills developed through the project.

3 Engineering Exploration: Required reflection after attendance at eight engineering related events that took place outside of the course. Events included seminars, engineering student group meetings, professional development activities, et cetera that were delivered by various groups within the university.
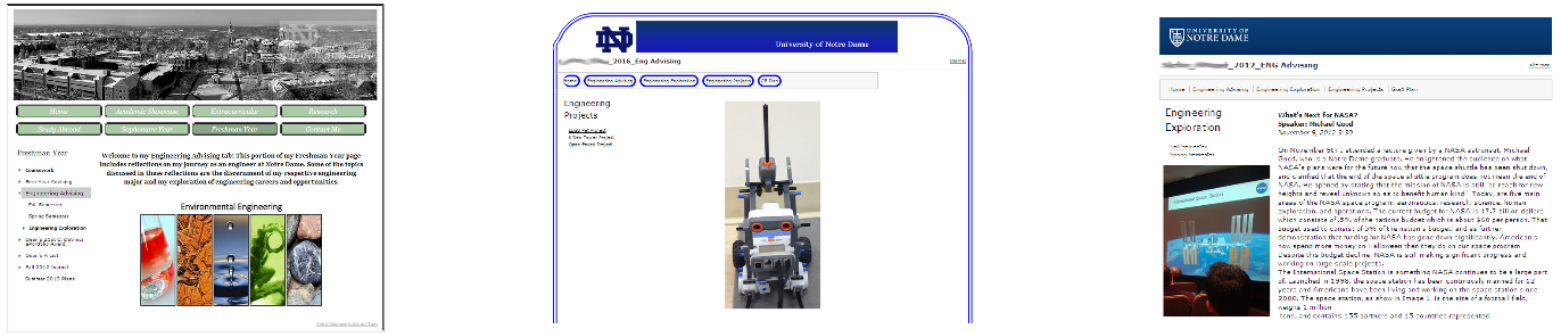

Figure 2: ePortfolio examples highlighting each of the three categories mentioned above.

Although ePortfolio assignments were a required portion of the course, they were graded largely for completion. Therefore, student effort towards their ePortfolio assignments had wide variability. In addition, students were encouraged to personalize their ePortfolios to include additional pages and information not required by the course. Because students were asked to share this ePortfolio with their advisors after matriculating into engineering departments, they were encouraged to keep any additional content professional in nature.

Goodrich et al. (2014) describes in detail how electronic portfolios have been incorporated into our Introduction to Engineering curriculum, and how they can potentially be used to measure student engagement. The paper contrasts instructor-generated ratings for student engagement with metrics extracted from those student ePortfolios, and it shows that for that particular cohort, the engagement estimates provided by the ePortfolio variables were significantly more strongly correlated to retention outcomes than were the instructor ratings. Given this particular context of how ePortfolios are utilized, we believe that an important correlation between the students' engagement in using this tool and retention levels exists and can be potentially mined for predictive analysis. 
(2014) Engagement vs Performance: Using Electronic Portfolios to Predict First Semester Engineering Student Persistence. Journal of Learning Analytics, 1 (3), 7-33.

\section{5 ePortfolio Use across the First Year}

At the time of this study, the University of Notre Dame was two years into a larger University ePortfolio initiative where half of the students on campus were using ePortfolios in at least one capacity. The College of First Year Studies had launched its own initiative for all first-year students, to flip and enhance the advising process of their one-on-one sessions with the ePortfolio. Using the blended advising model (Ambrose \& Williamson Ambrose, 2013), the Advising ePortfolio was used to pre-engage students by asking them to plan and list goals in their ePortfolio before their face-to-face advising session. This would lead to a more deeply engaged one-on-one advising interaction because the students came prepared. Then advisors and students would have a platform to re-engage and review progress and growth over the year. For a detail overview of this university-wide ePortfolio initiative, see Ambrose, Martin, and Page (2014).

In addition, a small cohort of 12 first-generation students (from a family in which no parent or guardian has earned a baccalaureate degree) who were intended Engineering students were also enrolled in a one-credit Independent Self-Study Advising Seminar as part of an invited scholars program for underrepresented students.

While section 5 will provide more detail on our datasets and describe each of the features we analyze, a preliminary case study illustrated by Figure 3 showed that this select group of students who were more exposed to electronic portfolios as part of the previously mentioned enhanced academic program exhibited markedly higher levels of engagement using that tool, and more importantly, was retained in its entirety. The figure below points to a possible transference and increased engagement effect when students are exposed to a more integrative, across time and other contexts, ePortfolio experience. In addition, it may also point to the engagement potential, particularly for a cohort of first-generation and underrepresented students.
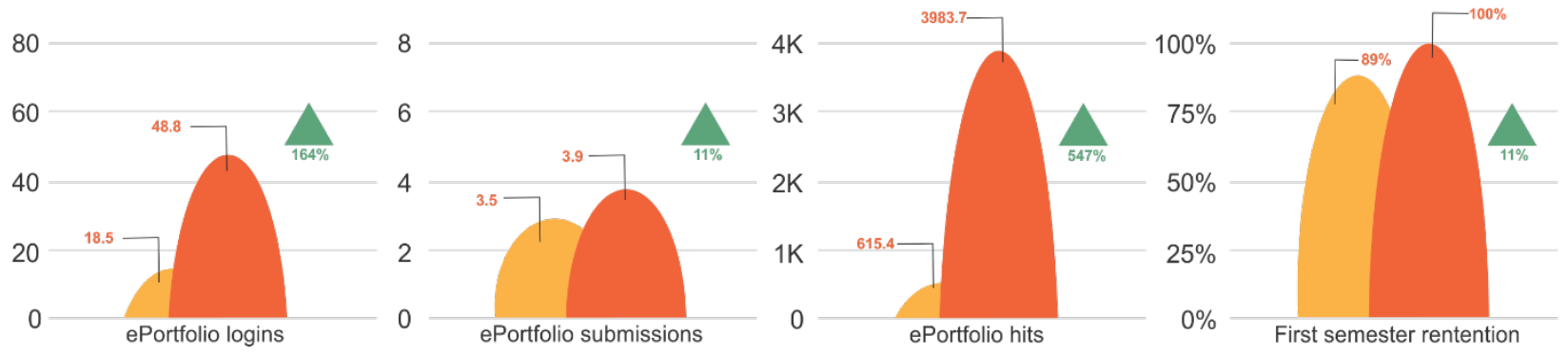

All first year engineering students

Select first generation students who were part of academic development program

Figure 3: The transference effect of ePortfolio engagement on a select first generation student sample. 
(2014) Engagement vs Performance: Using Electronic Portfolios to Predict First Semester Engineering Student Persistence. Journal of Learning Analytics, 1 (3), 7-33.

\section{PROBLEM, GOAL \& QUESTION}

As previously mentioned, factors providing early indications that a student may be at risk can come from a variety of categories. Data that has been aggregated over time can offer insights as to which subpopulations of a student body are more likely to need closer attention, whereas individual student-level performance data can be used to flag students who are beginning to diverge from their optimal path to success.

An important task, then, is to decide which information to track for each student so that we can most efficiently generate early risk warnings when these are warranted. As we claimed earlier, placing disproportional focus on academic performance data can result in warning systems that may fail to identify students losing interest and disengaging from school when these shortcomings are not reflected in that student's performance.

To illustrate, take for instance the performance data summarized in Figure 4. There we can see how the first semester cumulative GPAs for both retained and not retained students in our dataset were distributed. While it does appear that the retained students perform, on average, slightly better, we note that such disparity is not very substantial. Furthermore, we can see that a fairly sizable number of students who were not retained concluded their first semesters with very high GPAs (e.g., > 3.0), which is a reasonable indication that they would have been likely to succeed as engineering students had they chosen to remain enrolled in the program. A similar observation can be made when we look at the course grades for Introduction to Engineering (EG 111) alone.

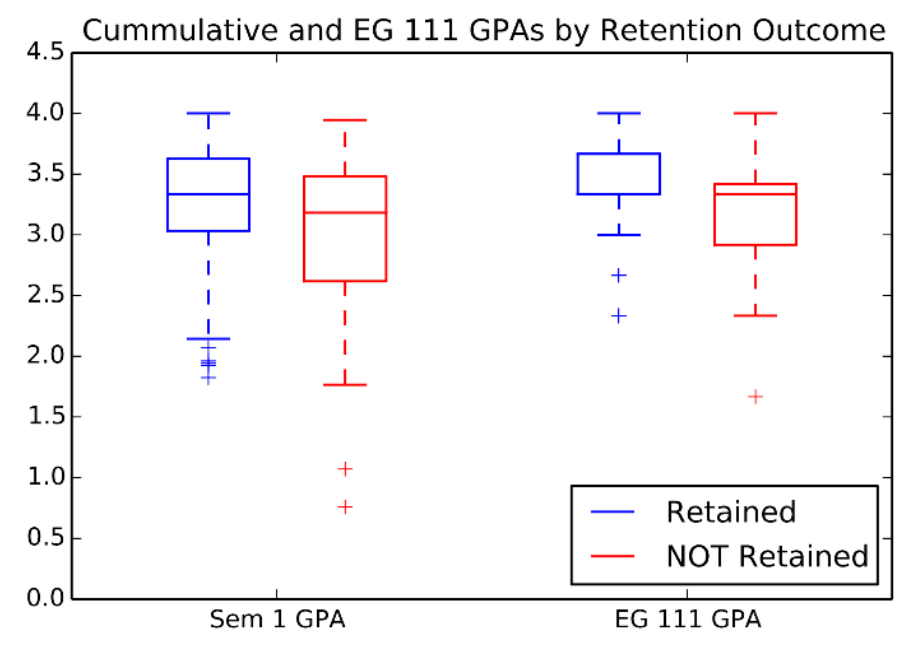

Figure 1: Academic performance breakdown by retention outcome. 
(2014) Engagement vs Performance: Using Electronic Portfolios to Predict First Semester Engineering Student Persistence. Journal of Learning Analytics, 1 (3), 7-33.

With that premise in mind, this paper attempts to investigate the viability of using ePortfolios as a proxy to measuring student engagement. Again, splitting the same cohort of students depicted in Figure 4 into the subgroups of retained and not retained, we see in Figure 5 that on average, students on each of these two groups appear to place discernibly different amounts of time and energy when interacting with their portfolios.
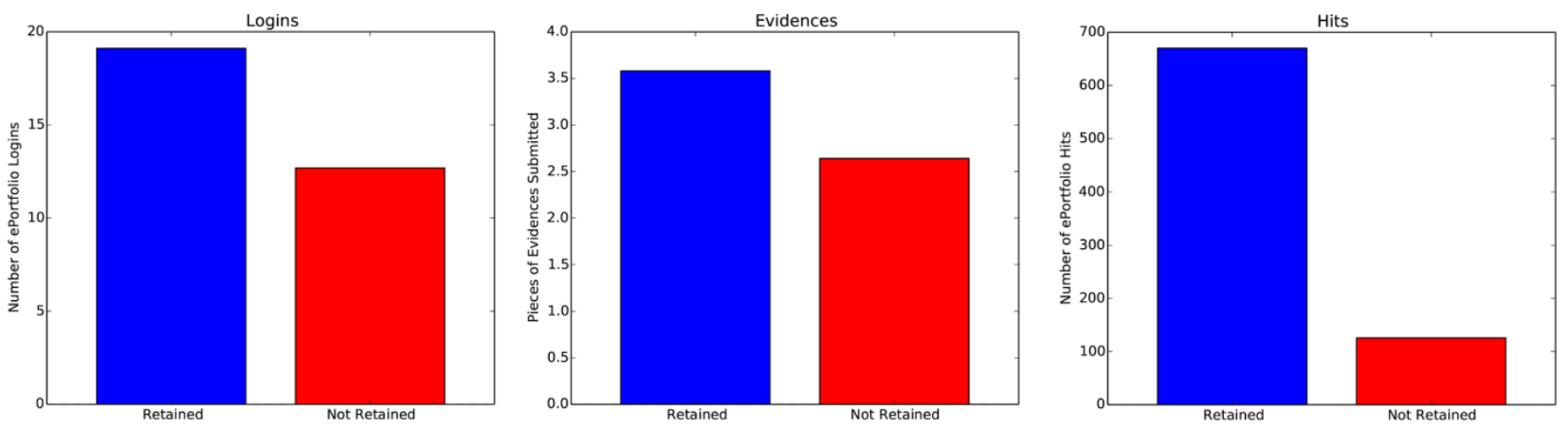

Figure 5: Average breakdown of ePortfolio engagement activity.

While Goodrich, Aguiar, Ambrose, Brockman, \& Chawla (2014) presents a more elaborate description of this information and a comparison between these observed values and similar engagement estimates generated by course instructors, we highlight that the distribution of values for each of the three features illustrated in Figure 5 (i.e., number of logins during the fall semester, number of evidences submitted through ePortfolio, and number of ePortfolio page hits received) with respect to retained and not retained students is statistically significantly different, with $p$-values displayed in Table 1.

Table 1: P-values of observed distributions for three ePortfolio features by retention outcomes.

\begin{tabular}{|c|c|}
\hline ePortfolio Feature & P-value \\
\hline Number of logins & $<0.005$ \\
\hline Number of evidences submitted & $<0.05$ \\
\hline Number of page hits & $<0.005$ \\
\hline
\end{tabular}


(2014) Engagement vs Performance: Using Electronic Portfolios to Predict First Semester Engineering Student Persistence. Journal of Learning Analytics, 1 (3), 7-33.

\section{DATASET}

This study used data collected from a single cohort of incoming freshmen students registered in a first semester Introduction to Engineering course. This particular group was made up of 429 students, the vast majority of whom had engineering majors listed as their first year intent and remained in the program for the subsequent semester, leading to a very imbalanced dataset. While majors are not formally declared until their sophomore year, students are asked to state their intended majors when submitting their application package and prior to their first semester on campus.

\subsection{Description}

A variety of features that describe each student's academic performance, engagement, and demographic background were made available to this project from multiple sources. These were then matched student-wise and merged into a single dataset. After an initial analysis of the data, we decided to exclude a number of features that either (1) had no apparent correlation to the outcome variable, (2) directly implied it, or (3) provided redundant information. Further, we also removed 10 instances that had a very considerable amount of missing data. These particular instances corresponded to students who dropped out early in the semester and hence had no academic performance or engagement data available. Table 2 lists and describes each feature available in our final dataset and Table 3 groups these into their respective categories.

Table 2: Dataset features described

\begin{tabular}{|c|c|c|}
\hline Name & Description & Type \\
\hline Adm Intent & $\begin{array}{l}\text { Intended college major as specified by the student in his/her application } \\
\text { package }\end{array}$ & Nominal \\
\hline Adm Type & Type of admission earned by student (e.g., early, regular, waiting list) & Nominal \\
\hline AP Credits & Number of credits earned through AP courses taken prior to college enrollment & Numeric \\
\hline Dormitory & $\begin{array}{l}\text { Name of dorm where the student resides (all first-year students are required to } \\
\text { live on campus) }\end{array}$ & Nominal \\
\hline EG 111 Grade & Letter grade obtained in the introduction to engineering course & Numeric \\
\hline ePort Hits & Hit count for the student's ePortfolio pages during the fall semester & Numeric \\
\hline ePort Logins & $\begin{array}{l}\text { Number of times the student logged in to his/her ePortfolio account during the } \\
\text { fall semester }\end{array}$ & Numeric \\
\hline ePort Subm & Number of assignment submitted via ePortfolio during the fall semester & Numeric \\
\hline Ethnicity & The student's self-declared ethnicity & Nominal \\
\hline
\end{tabular}


(2014) Engagement vs Performance: Using Electronic Portfolios to Predict First Semester Engineering Student Persistence. Journal of Learning Analytics, 1 (3), 7-33.

\begin{tabular}{|c|c|c|}
\hline First Gen & A flag to denote first-generation college students & Binary \\
\hline FY Intent & $\begin{array}{l}\text { Intended college major as specified immediately prior to the beginning of the } \\
\text { fall semester }\end{array}$ & Nominal \\
\hline Gender & The student's gender & Binary \\
\hline $\begin{array}{l}\text { Income } \\
\text { Group }\end{array}$ & $\begin{array}{l}\text { A numeric value ranging from } 1-21 \text {, each corresponding to a different income } \\
\text { group segment }\end{array}$ & Numeric \\
\hline SAT Comb & Combined SAT scores & Numeric \\
\hline SAT Math & SAT score for the math portion of the test & Numeric \\
\hline SAT Verbal & SAT score for the verbal portion of the test & Numeric \\
\hline Sem 1 GPA & The student's overall GPA at the end of the fall semester & Numeric \\
\hline Retained & $\begin{array}{l}\text { A flag identifying students that left the program immediately after the fall } \\
\text { semester }\end{array}$ & Binary \\
\hline
\end{tabular}

It is worth noting that this particular dataset has a highly imbalanced class distribution wherein only 11.5 percent of the instances belong to the minority class (student not retained). As described in Thammasiri et al. (2013), predicting student retention becomes more challenging when the available training sets are imbalanced because standard classification algorithms usually have a bias towards the majority class.

\subsection{Feature Selection}

As a second step to preparing our dataset, we carried out a series of tests to investigate how strongly correlated to the outcome each feature was. In general, performing feature selection as a means for reducing the feature space provides some benefits when building classification models. Namely, the model becomes more generalizable and less prone to overfitting, more computationally efficient, and easier to interpret.

The following feature selection methods were used: information gain (IG) (Quinlan, 1986), gain ratio (GR) (Quinlan, 1993), chi-squared (CS), and Pearson's correlation (CR). The first evaluates the worth of each attribute by measuring its information gain with respect to the class. Gain ratio works in a similar manner while adopting a different metric. CS and CR compute chi-squared and Pearson's correlation statistics for each feature/class combination.

The results of our experiments are summarized in Table 4, where the top 10 features ranked by each method are listed, and the highest correlated one is highlighted for each column. 
(2014) Engagement vs Performance: Using Electronic Portfolios to Predict First Semester Engineering Student Persistence. Journal of Learning Analytics, 1 (3), 7-33.

Table 1: Dataset features categorized.

\begin{tabular}{|c|c|c|}
\hline Type & Subtype & Feature \\
\hline \multirow{9}{*}{ Academic } & \multirow{5}{*}{ Pre-Adm } & Adm Intent \\
\hline & & SAT Math \\
\hline & & SAT Verbal \\
\hline & & SAT Comb \\
\hline & & AP Credits \\
\hline & \multirow{4}{*}{ Post-Adm } & FY Intent \\
\hline & & EG 111 Grade \\
\hline & & Sem 1 GPA \\
\hline & & Retained \\
\hline \multirow{6}{*}{ Demographics } & & Adm Type \\
\hline & & Gender \\
\hline & & Ethnicity \\
\hline & & Income Group \\
\hline & & First Gen \\
\hline & & Dormitory \\
\hline \multirow{3}{*}{ Engagement } & & ePort Logins \\
\hline & & ePort Subm \\
\hline & & ePort Hits \\
\hline
\end{tabular}

Table 4: Feature selection rank.

\begin{tabular}{|c|c|c|c|c|}
\hline & IG & GR & CS & CR \\
\hline Adm Intent & 2 & 4 & 2 & - \\
\hline SAT Math & 7 & 2 & 5 & 3 \\
\hline SAT Verbal & - & - & - & - \\
\hline SAT Comb & 9 & 10 & 9 & - \\
\hline AP Credits & 8 & 8 & 8 & 8 \\
\hline FY Intent & 5 & 7 & 6 & 9 \\
\hline EG 111 Grade & 4 & 5 & 3 & 6 \\
\hline Sem 1 GPA & - & - & - & 1 \\
\hline Adm Type & - & - & - & - \\
\hline Gender & 10 & 9 & 10 & 5 \\
\hline Ethnicity & - & - & - & - \\
\hline Income Group & - & - & - & 7 \\
\hline First Gen & - & - & - & - \\
\hline Dormitory & 3 & 6 & 4 & - \\
\hline ePort Logins & 6 & 3 & 7 & 4 \\
\hline ePort Subm & - & - & - & 10 \\
\hline ePort Hits & 1 & 1 & 1 & 2 \\
\hline
\end{tabular}

Several interesting observations can be derived from these results. First, we emphasize that all but one method reported ePort Hits as being the most important feature of the dataset. In other words, there appears to be a strong correlation between the number of times a certain student's electronic portfolio pages are visited and that student's decision to stay or withdraw from the College of Engineering. Note that these hits originate from both external visitors and the students themselves. While the current data does not allow us to discern the two scenarios, we suspect that the majority of the hits do in fact come from the portfolio owner. If that is indeed the case, this noticeable correlation could be explained simply by the fact that students whose portfolios exhibit a larger number of hits are likely to be those who spend more time editing their pages, creating new content, and submitting assignments (as those actions directly contribute to that student's page hit count). It would then make reasonable sense that 
(2014) Engagement vs Performance: Using Electronic Portfolios to Predict First Semester Engineering Student Persistence. Journal of Learning Analytics, 1 (3), 7-33.

this display of engagement should, in some cases, correlate with the chances of this particular student being retained.

Further, we noticed that some of the features had no substantial individual correlation to the class values. For instance, in our particular context, ethnicity, admission type, first generation status, income, and the number of assignments a student sent through his/her ePortfolio did not appear to be closely related to that student's retention in the program. As reported by Zhang, Anderson, Ohland, and Thorndyke (2004) and by Mendez, Buskirk, Lohr, and Haag (2008), we also observed minor negative correlations between verbal SAT scores and engineering student retention.

To compare the performance of classification models based on traditional academic data to that of models based on student engagement features effectively, we created four subsets from the original data. These are described below:

- All-academic: This subset contained all academic and demographic features listed in Table 3.

- Top-academic: Following the feature selection process described above, this subset contains only the top three academic and demographic features. Multiple wrapper methods (i.e., which can score feature subsets rather than individual features alone) were used, and the final subset chosen contained the following: Admin intent, EG 111 grade, and Sem 1 GPA.

- All-engagement: Contained the three ePortfolio engagement features.

- Top-academic + engagement: This final subset contained the optimal three-element combination of features across all initially available. These were EG 111 grade, ePort logins, and ePort hits.

\section{METHODOLOGY}

For this study, we selected a range of classification methods that have been previously utilized in this particular domain, or that are suitable to work with imbalanced datasets. Following is a brief description of each classifier and the evaluation measurements we use to compare their performance.

\subsection{Classification Methods}

\subsubsection{Naïve Bayes}

Among the simplest and most primitive classification algorithms, this probabilistic method is based on the Bayes Theorem (Bayes \& Price, 1763) and strong underlying independence assumptions. That is, each feature is assumed to contribute independently to the class outcome. In predicting student attrition, naïve Bayes classifiers have been used by Pittman (2011), Zhang, Oussena, Clark, and Hyensook (2010), and Nandeshwar, Menzies, and Nelson (2011). Notably, the best results reported in Zhang et al. (2010) were achieved via this method. 
(2014) Engagement vs Performance: Using Electronic Portfolios to Predict First Semester Engineering Student Persistence. Journal of Learning Analytics, 1 (3), 7-33.

\subsubsection{C4.5 Decision trees}

Another very popular classification method, C4.5 decision trees (Quinlan, 1993) have been used to predict student retention multiple times in recent literature such as in Yadav, Bharadwaj, and Pal (2012), Nandeshwar, Menzies, and Nelson (2011), Lauría (2012), and Lin (2012). This method works by building a tree structure where split operations are performed on each node based on information gain values for each feature of the dataset and the respective class. At each level, the attribute with highest information gain is chosen as the basis for the split criterion.

\subsubsection{Logistic regression}

Logistic regression is often used as a classification method wherein a sigmoid function is estimated based on the training data, and used to partition the input space into two class specific regions. Given this division, new instances can be easily verified to belong to one of the two classes. This approach has been used to predict student retention, and has often achieved highly accurate results (Luna, 2000; Fike \& Fike, 2008; Lauría, 2012; Lin, Imbrie, \& Reid, 2009; Zhang, Anderson, Ohland, \& Thorndyke, 2004; Herzog, 2006; Veenstra, Dey, \& Herrin, 2009).

\subsubsection{Hellinger distance decision trees}

When applying learning algorithms to imbalanced datasets, one often needs to supplement the process with some form of data sampling technique. Hellinger distance decision trees were proposed as a simpler alternative to that (Cieslak, Hoens, Chawla, \& Kegelmeyer, 2012). This method uses Hellinger distance as the splitting criterion for the tree, which has several advantages over traditional metrics, such as gain ratio in the context of imbalanced data. To the best of our knowledge, this method has not yet been used to predict student retention, but given that our dataset is highly imbalanced, we chose to investigate its performance.

\subsubsection{Random forests}

Random forests combine multiple tree predictors in an ensemble (Breiman, 2001). New instances being classified are pushed down the trees, and each tree reports a classification. The "forest" then decides which label to assign to this new instance based on the aggregate number of votes given by the set of trees. Recent work by Mendez, Buskirk, Lohr, and Haag (2008) used this method to predict science and engineering student persistence.

\subsection{Evaluation Measures}

In order to compare the results obtained by each of the classifiers, as well as the four different data "subsets," we utilized a variety of measures. A very popular standard used to evaluate classifiers is the predictive accuracy. Note, however, that utilizing this metric to evaluate classification based on imbalanced datasets can be extremely misleading. 
(2014) Engagement vs Performance: Using Electronic Portfolios to Predict First Semester Engineering Student Persistence. Journal of Learning Analytics, 1 (3), 7-33.

To illustrate this issue, suppose that upon being given our dataset, an imaginary classifier predicts that all students will be retained in the engineering program following their first semester enrolled on campus. This will result in a remarkable 88.5 percent accuracy (recall that only 11.5 percent of the students in this dataset left the program at the end of their first semester). It is obvious, however, that such a classifier should not be awarded any merit since it fails to identify all students that should have been labelled as being at risk. Instead, it is more appropriate to analyze the prediction accuracy for each individual class, or to use ROC curves to summarize the classifier performance. These and other measures can be calculated using confusion matrices (see Figure 6).

Given the binary nature of this specific classification problem, the corresponding confusion matrix reports four values: True Positives (TP) - the number of students not retained correctly classified; True Negatives (TN) - the number of retained students accurately classified as such; False Positives (FP) the number of retained students mistakenly classified as not retained; and False Negatives (FN) - not retained students wrongfully predicted as retained. Based on these labels, the individual accuracies for the negative (retained) and positive ${ }^{2}$ (not retained) classes, as well as the classifier's recall rates can be obtained as follows:

$$
\begin{aligned}
\text { accuracy }^{+} & =\frac{T P}{T N+F N} \\
\text { accuracy }^{-} & =\frac{T N}{T N+F N} \\
\text { recall } & =\frac{T P}{T P+F N}
\end{aligned}
$$

As previously mentioned, ROC curves are frequently used to summarize the performance of classifiers on imbalanced datasets. On an ROC curve, the $\mathrm{x}$-axis represents the FP rate, $F P /(T N+F P)$, and the $\mathrm{y}^{-}$ axis denotes the TP rate given by $T P /(T P+F N)$ at various threshold settings. The area under the ROC curve, AUROC, is also a useful metric for comparing different classifiers. The values for AUROC range from a low of 0 to a high of 1 , which would represent an optimal classifier, as highlighted in Figure 7.

${ }^{2}$ The prediction accuracy for the positive class can also be labelled precision. 
(2014) Engagement vs Performance: Using Electronic Portfolios to Predict First Semester Engineering Student Persistence. Journal of Learning Analytics, 1 (3), 7-33.

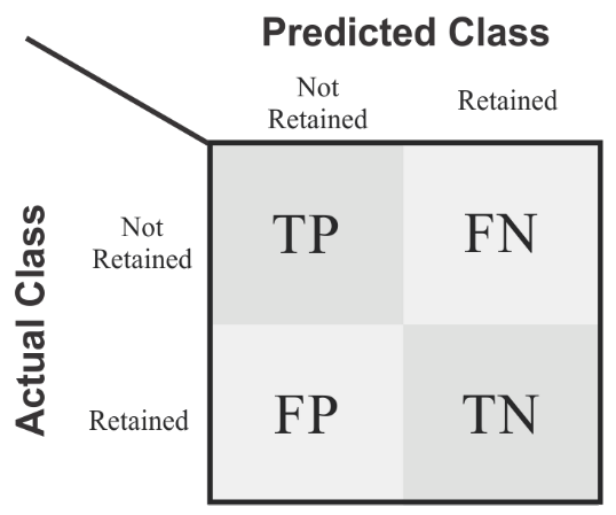

Figure 6: Confusion matrix for our experiments

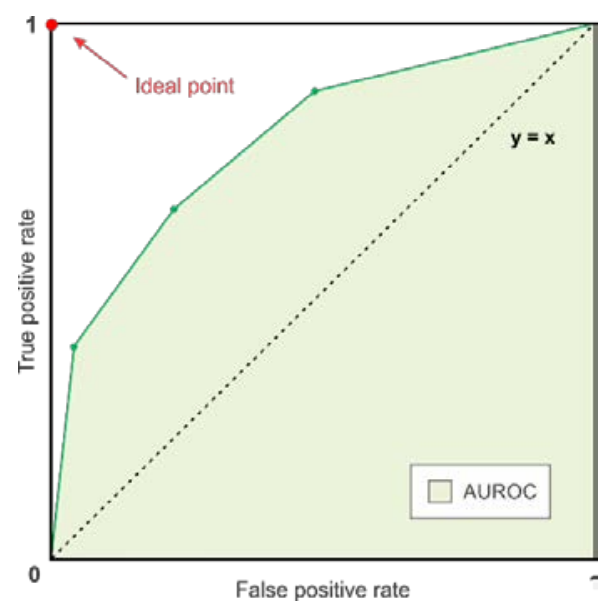

Figure 2: ROC curve illustration

\section{EXPERIMENTAL RESULTS}

To estimate how well the models generalize to future datasets, we utilized a 10 -fold cross validation technique. This consists of splitting the original $n$ data instances into 10 complementary subsets of size $n / 10$, each of which preserving the original ratio of minority and majority class instances. The classifier is then given 9 of the subsets for training, and validation is performed using the remaining portion of the data. This process is repeated for 10 rounds using different partitions at each time, and an overall average of the results across each iteration is computed.

The performance of each of the five classification methods described in section 6.1 was evaluated as they were used to perform prediction on each of the four available datasets. Table 4 displays the results of each individual experiment in terms of the prediction accuracy for the negative class instances (i.e., the ratio of retained students correctly labelled as retained), the prediction accuracy for the positive 
(2014) Engagement vs Performance: Using Electronic Portfolios to Predict First Semester Engineering Student Persistence. Journal of Learning Analytics, 1 (3), 7-33.

class instances (i.e., the ratio of not retained students correctly classified as not retained), and the overall weighted average across these two accuracy measurements. The highest accuracies achieved for each of the datasets are highlighted in bold, while the three highest overall are underlined.

Before analyzing these results more deeply, it is essential to consider the degree of importance that should be assigned to each of these metrics. Given our binary classification problem, two types of errors could emerge. Students who ultimately remain in the program for the spring semester could be misclassified as not retained (false positives), and actual not-retained students could be mistakenly labelled as retained (false negatives). While some previous work (Dekker, Pechenizkiy, \& Vleeshouwers, 2009) considered the first type of error to be more serious, we argue that the opposite is true. If these techniques are to be used in the development of an effective early warning system, failing to identify students at risk of leaving can be much more costly than incorrectly labelling someone as an early leaver.

In Table 5, we can see that predictions based only on academic performance and demographic data achieve a maximum acc+ of 27.5 percent when the all-academic dataset is paired with a naïve Bayes model, which corresponds to only 11 of the 48 not-retained students being correctly identified. Conversely, when engagement features are utilized, that accuracy improves very noticeably to 83.3 percent and 87.5 percent, both also achieved with the previously mentioned classifier.

The naïve Bayes model, using the top-academic + engagement dataset, remarkably identified 42 of the 48 dropout students. The vast majority of those retained (331 out of 419) are also correctly classified. Note that the other four classifiers obtain higher acc-values under the same setup, and could potentially be the preferred choice depending on the circumstances.

With respect to acct, the naïve Bayes classifier outperformed the others for all but one dataset. We used its experimental results in Figure 8 to illustrate the ROC and Precision-Recall curves for each dataset. In our particular context, it seems apparent that the ePortfolio engagement features are very good predictors for student retention. The highest AUROC value ${ }^{3}(0.929)$ was obtained by the topacademic + engagement dataset, while all-academic performed worse with an AUROC of 0.654 .

\footnotetext{
${ }^{3}$ A different logistic regression implementation using L1 regularization yielded slightly higher AUROC. See Table 5 for details. ISSN 1929-7750 (online). The Journal of Learning Analytics works under a Creative Commons License, Attribution - NonCommercial-NoDerivs 3.0 Unported (CC BY-NC-ND 3.0) 24
} 
(2014) Engagement vs Performance: Using Electronic Portfolios to Predict First Semester Engineering Student Persistence. Journal of Learning Analytics, 1 (3), 7-33.

Table 5: Prediction accuracy achieved using each of the datasets

\begin{tabular}{|c|c|c|c|c|}
\hline Dataset & Classifier & Acct & Acc- & Acc \\
\hline \multirow{5}{*}{ all-academic } & NB & 0.275 & 0.902 & 0.842 \\
\hline & DT & 0.083 & 0.930 & 0.833 \\
\hline & LR & 0.104 & 0.900 & 0.803 \\
\hline & HT & 0.083 & 0.884 & 0.792 \\
\hline & RF & 0.000 & $\underline{0.987}$ & 0.874 \\
\hline \multirow{5}{*}{ top-academic } & NB & 0.167 & 0.954 & 0.864 \\
\hline & DT & 0.042 & 0.949 & 0.845 \\
\hline & LR & 0.000 & 0.981 & 0.869 \\
\hline & HT & 0.250 & 0.881 & 0.809 \\
\hline & RF & 0.104 & 0.892 & 0.802 \\
\hline \multirow{5}{*}{ all-engagement } & NB & 0.833 & 0.879 & 0.874 \\
\hline & DT & 0.771 & 0.970 & 0.947 \\
\hline & LR & 0.771 & 0.978 & $\underline{0.955}$ \\
\hline & HT & 0.771 & 0.962 & 0.940 \\
\hline & RF & 0.771 & 0.970 & 0.947 \\
\hline \multirow{5}{*}{ top-academic+engagement } & NB & $\underline{0.875}$ & 0.892 & 0.890 \\
\hline & DT & 0.792 & 0.962 & 0.945 \\
\hline & LR & 0.750 & 0.973 & 0.947 \\
\hline & HT & 0.771 & 0.965 & 0.943 \\
\hline & $\mathrm{RF}$ & 0.750 & 0.965 & 0.940 \\
\hline
\end{tabular}


(2014) Engagement vs Performance: Using Electronic Portfolios to Predict First Semester Engineering Student Persistence. Journal of Learning Analytics, 1 (3), 7-33.
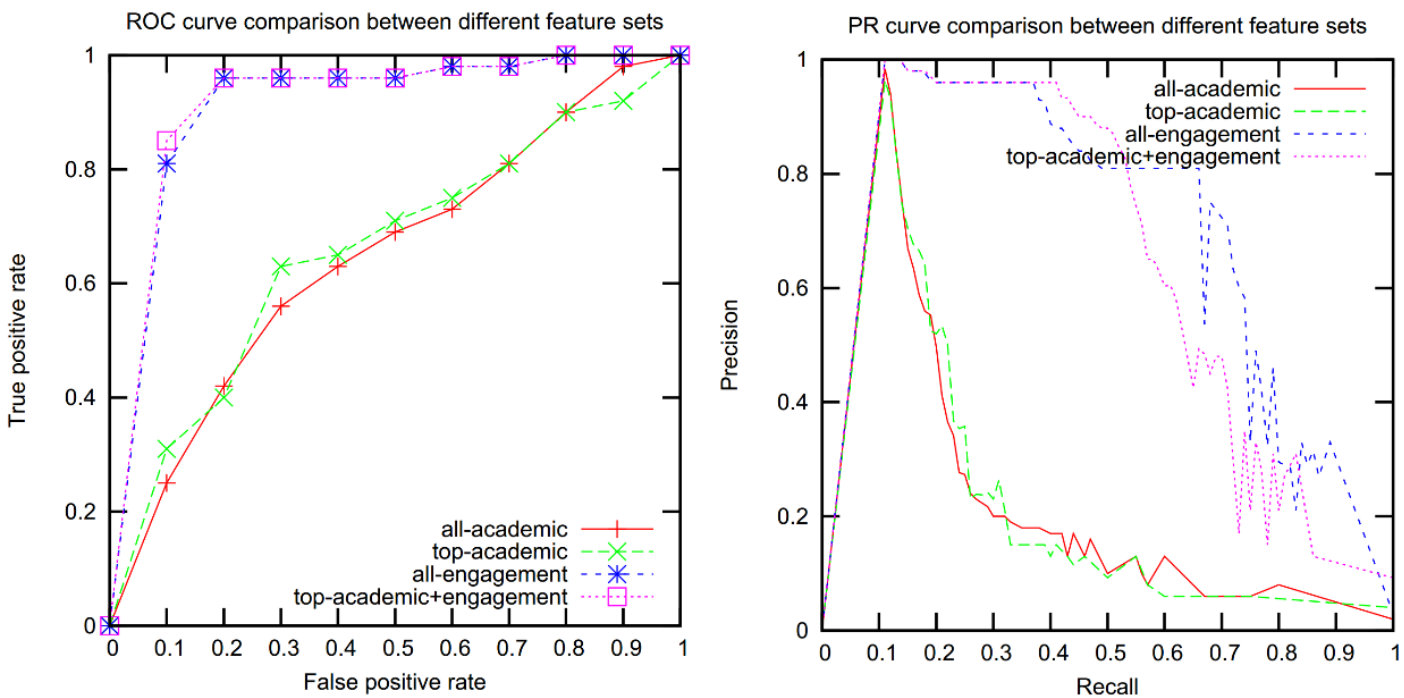

Figure 8: Naive Bayes ROC and Precision-Recall

\subsection{Improving the Detection of at-risk Students with Data Oversampling}

As highlighted in section 5.1, the particular cohort of students utilized for our experiments had a very high retention rate of 88.5 percent. With such data imbalance, classification models can often learn hypothesis that are biased towards assigning the majority class label to new instances. One way to address this issue is to introduce, during the training phase of the models, "synthetic" examples of minority class instances. This can essentially decrease the rate of imbalance and it often lessens the bias towards the majority class. Previous work by Thammasiri et al., (2013) has investigated the effect of various over-sampling techniques on models designed to predict student attrition. One such method that was found to improve the overall performance was SMOTE (Chawla, Bowyer, Hall, \& Kegelmeyer, 2002), which works by generating synthetic instances by interpolating existing minority class occurrences with their k-nearest neighbours of the same class.

To evaluate any benefits of data oversampling in our context, we used the top-academic + engagement dataset previously described, and repeated the experiments while introducing synthetic instances of the not-retained class. Table 5 illustrates the performances of our models in terms of AUROC when varying amounts of synthetic minority instances are used. There, "100 SMOTE" refers to the addition of a synthetic set of minority instances that contains the same number of samples as the original set, "200 SMOTE" creates two new samples for each observed minority instance, and so on. As we can see, most models exhibit marginal improvements when SMOTE instances are used to decrease the class imbalance during model training. 
(2014) Engagement vs Performance: Using Electronic Portfolios to Predict First Semester Engineering Student Persistence. Journal of Learning Analytics, 1 (3), 7-33.

Table 6: Classification models' AUROC with data

\begin{tabular}{|c|c|c|c|c|}
\hline Classifier & No SMOTE & 100 SMOTE & 200 SMOTE & 300 SMOTE \\
\hline NB & 0.923 & 0.923 & 0.924 & $\mathbf{0 . 9 2 6}$ \\
\hline DT & 0.858 & $\mathbf{0 . 8 6 6}$ & 0.855 & 0.849 \\
\hline LR & 0.968 & 0.969 & 0.970 & $\underline{\mathbf{0 . 9 7 1}}$ \\
\hline RF & 0.953 & $\mathbf{0 . 9 6 0}$ & 0.956 & 0.941 \\
\hline
\end{tabular}

\subsection{Alternative Model Evaluation}

While the previous sections provided a detailed comparison between the performances obtained by each model with respect to a variety of traditionally used evaluation metrics, when considering the realworld requirements of implementing an early warning system wherein interventions are put in place to help students labelled as being at risk, it is often more logical to evaluate our predictive models differently. Suppose for instance that a school is given a certain operational budget to deploy an intervention program for STEM students at risk of not being retained. Given this particular constraint, it might not be possible for the school administration to hold individual one-on-one interventions with all students at risk. Hence, they should ideally choose a predictive model that can most accurately identify which students are at highest risk.

The models described in section $6.1 \mathrm{can}$, in addition to providing a binary label to a new student being evaluated, also output an associated probability value between 0 and 1 that corresponds to that model's estimate of the likelihood of this student not being retained. Based on a set threshold value in that same range, the model then decides if the student is to be labelled as likely to be retained or otherwise. Contextually, if a predictive model using a threshold of 0.5 generates a probability of 0.6 for student $A$ and 0.99 for student $B$, they will both receive a "not retained" label. However, chances are that student $B$ requires more immediate attention.

If we generate a probability value for each student in a given cohort, we can then create an overall sorted rank with respect to these values. In this setting, a preferred model would be one that can generate high probabilities for those students indeed at risk and lower probabilities for those on track to succeeding. Next, we compare the performances of four of our models in terms of how precise they are on the top 5 and 10 percent of the probability scores they generate. ${ }^{4}$ That is, if we isolate the 5 and 10 percent of students who receive the highest scores from each model, we would like to know what fraction of that group eventually left engineering. This metric is especially helpful in the context of early warning systems because when working with time and budgetary constraints, one will often need to choose a small subset of students on which to focus the appropriate intervention.

${ }^{4}$ A 10 -fold cross validation setup was utilized to score all students. 
(2014) Engagement vs Performance: Using Electronic Portfolios to Predict First Semester Engineering Student Persistence. Journal of Learning Analytics, 1 (3), 7-33.

As seen in Table 6, the models that include engagement features outperform those that only consider academic performance when we look at precisions on both the top 5 and 10 percent. Further, we highlight that the results obtained by the logistic regression model that only used engagement features were the highest observed. In our specific scenario, the top 5 percent accounted for the 22 students whose probability scores were highest overall. Using a logistic regression (or decision tree) model coupled with the engagement dataset to select the 22 students at "highest risk" gives us a precision of 0.954 , which means that 21 of the 22 selected students were in fact not retained.

When pairing at-risk students with the appropriate interventions based on flags generated by early warning systems, we might not necessarily give preference to models that can be highly accurate, but we should rather evaluate their ability to select those students who need the most immediate attention. Though we had previously shown that the naïve Bayes model is the most accurate in the aggregate, here we show that other models, in fact, might provide a more appropriate solution from an operational standpoint.

Table 7: Precision on the top 5 and $10 \%$

\begin{tabular}{|c|c|c|c|}
\hline Dataset & Classifier & Top 5\% & Top $10 \%$ \\
\hline \multirow{4}{*}{ all-academic } & NB & 0.400 & 0.268 \\
\hline & DT & 0.200 & 0.243 \\
\hline & LR & 0.400 & 0.341 \\
\hline & RF & 0.350 & 0.292 \\
\hline \multirow{4}{*}{ top-academic } & NB & 0.300 & 0.268 \\
\hline & DT & 0.250 & 0.244 \\
\hline & LR & 0.400 & 0.268 \\
\hline & RF & 0.150 & 0.170 \\
\hline \multirow{4}{*}{ all-engagement } & NB & 0.800 & 0.756 \\
\hline & DT & $\underline{0.954}$ & 0.756 \\
\hline & LR & $\underline{0.954}$ & $\underline{0.878}$ \\
\hline & RF & 0.900 & 0.829 \\
\hline \multirow{4}{*}{ top-academic+engagement } & NB & 0.650 & 0.682 \\
\hline & DT & 0.900 & 0.756 \\
\hline & LR & 0.900 & 0.878 \\
\hline & RF & 0.900 & 0.829 \\
\hline
\end{tabular}


(2014) Engagement vs Performance: Using Electronic Portfolios to Predict First Semester Engineering Student Persistence. Journal of Learning Analytics, 1 (3), 7-33.

\section{CONCLUSION AND FUTURE WORK}

Given a course with an enrollment of 429 first-semester, first-year Intro to Engineering students and an average retention rate of 85 percent, the challenge was to develop, train, and test classification models to predict whether students would persist into the second semester beyond traditional measures of performance. How do you identify not just the high interest/low ability at risk students but also the low interest/high ability students? More specifically, we wanted to compare the performances of models based on traditional academic data (SAT scores, GPA, demographics, etc.) and models based on data extracted from ePortfolios (\#logins, \#hits, etc.). The basic premise of this being that while early warning systems based on academic performance data alone can be very good at finding students struggling in class and alerting their advisors, early signs of a possible decision to drop out of a certain academic path can be very difficult to find when you are dealing with students who do not display any changes in their class performance.

We investigated the feasibility of using ePortfolio data as a proxy to measuring student engagement and showed that these particular variables can be highly predictive of college retention outcomes. We described that while datasets that do not contain features that quantify student academic engagement can often yield reasonable results, providing such features to the classification models greatly increases their ability to identify students who may ultimately leave. Our experiments showed significant gains in accuracy when engagement features were utilized, and we believe this can be used to build early warning systems that would be able to identify at-risk students at very early stages of their academic life, giving educators the opportunity to intervene in a more timely and effective fashion. Our key findings included the following:

- Out of a set of several academic performance, demographic, and ePortfolio features, the number of ePortfolio hits displayed, based on multiple metrics, the strongest correlation values to outcome (student was retained/not retained).

- The performance of the prediction models that used only ePortfolio data was consistently better than that of models based on academic performance data alone.

- The best prediction results were obtained by using a subset of the data containing the following features: EG 111 grade, ePort logins, and ePort hits.

- Using only academic performance data and a leave-one-out cross validation setup, we were able to identify 11 of the 48 students not retained past their first semester (out of the 429 students in the course). By adding the ePortfolio features, our model's performance dramatically improved and we were able to label 42 of the 48 students correctly while incurring very minor losses in accuracy regarding the retained group.

While the results presented in this paper were obtained using an aggregated dataset collected at the end of the fall semester of 2012, we have since begun collecting ePortfolio usage data on a daily basis. As we have shown for this particular cohort of engineering students, low engagement with this tool can 
(2014) Engagement vs Performance: Using Electronic Portfolios to Predict First Semester Engineering Student Persistence. Journal of Learning Analytics, 1 (3), 7-33.

work as an indicator that a certain student might not persist in engineering coursework in the following semester. With that in mind, we plan to investigate how soon after the beginning of the semester one can effectively determine that a student is "disengaging" and what interventions can be conducted during the semester to attempt to increase engagement levels before the course ends.

Furthermore, we have begun to compare, combine, and triangulate traditional learning analytics data from Sakai, the campus learning management system, with the academic, demographic, and engagement data sets. Other programs in the College of Science are also attempting to replicate and scale the ePortfolio design, utilization, and analytics gained from this Intro to Engineering course.

While for the most part we can measure how much time and energy a student puts into his/her ePortfolio by looking at the quantitative metrics discussed in this paper, there may also be substantial knowledge to be gained from understanding the qualitative data within the ePortfolio. To that end, we have also started to explore insights that can be harvested by text mining the actual content of students' ePortfolios. Lastly, we intend to condense this predictive analysis and students' engagement trajectories over time into an interactive dashboard that can be used by academic advisors who will then be able to track student progress and decide who is in need of individual attention and when that intervention should take place.

\section{ACKNOWLEDGEMENTS}

We thank Jeffrey Yan and Peter Lefferts at Digication for the help they provided with the collection of the ePortfolio datasets, and Catherine Pieronek for compiling the academic performance data.

\section{REFERENCES}

Accreditation Board for Engineering and Technology. (2013, 10 16). Retrieved from http://www.abet.org Al-Atabi, M., Mahdi, A. S., Younis, O., \& Chung, E. (2011). An integrated portfolio and advising system for undergraduate engineering students. Journal of Engineering Science and Technology, 6(5), 532541.

Alkhasawneh, R. (2011, July). Developing a Hybrid Model to Predict Student First Year Retention and Academic Success in STEM Disciplines Using Neural Networks. Ph.D. dissertation, Virginia Commonwealth University.

Ambrose, G. A., \& Williamson Ambrose, L. (2013). The Blended Advising Model: Transforming Advising with ePortfolios. International Journal of ePortfolio, 3(1), 75-89.

Ambrose, G. A., Martin, H., \& Page, H. (2014). Linking Advising and ePortfolios for Engagement: Design, Evolution, Assessment, \& University Wide Implementation. AAC\&U Peer Review Winter.

Astin, A. W., \& Astin, H. S. (1992). Undergraduate Science Education: The Impact of Different College Environments on the Educational Pipeline in the Sciences. Final Report. 
(2014) Engagement vs Performance: Using Electronic Portfolios to Predict First Semester Engineering Student Persistence. Journal of Learning Analytics, 1 (3), 7-33.

Barrett, H. C. (2007). Researching electronic portfolios and learner engagement: The REFLECT initiative. Journal of Adolescent \& Adult Literacy, 50(6), 436-449.

Bayes, M., \& Price, M. (1763). An Essay towards solving a Problem in the Doctrine of Chances. By the late Rev. Mr. Bayes, communicated by Mr. Price, in a letter to John Canton, M.A. and F.R.S. Philosophical Transactions (1683-1775), 370-418.

Breiman, L. (2001). Random forests. Machine learning, 45(1), 5-32.

Burtner, J. (2005). The Use of Discriminant Analysis to Investigate the Influence of Non-Cognitive Factors on Engineering School Persistence. Journal of Engineering Education, 94(3), 335-338.

Chawla, N. V., Bowyer, K. W., Hall, L. O., \& Kegelmeyer, W. P. (2002). SMOTE: Synthetic Minority Oversampling Technique. Journal of Artificial Intelligence Research, 16, 321-357.

Chen, H. L., \& Black, T. C. (2010). Using E-Portfolios to Support an Undergraduate Learning Career: An Experiment with Academic Advising. Educause Quarterly, 33(4).

Cieslak, D. A., Hoens, T. R., Chawla, N. V., \& Kegelmeyer, W. P. (2012). Hellinger distance decision trees are robust and skew-insensitive. Data Mining and Knowledge Discovery, 24(1), 136-158.

Davis, J., \& Goadrich, M. (2006). The relationship between Precision-Recall and ROC curves. Proceedings of the 23rd international conference on Machine learning, (pp. 233-240).

DeBerard, M. S., Spielmans, G., \& Julka, D. (2004). Predictors of academic achievement and retention among college freshmen: A longitudinal study. College Student Journal, 38(1), 66-80.

Dekker, G., Pechenizkiy, M., \& Vleeshouwers, J. (2009). Predicting Students Drop Out: A Case Study. International Conference on Educational Data Mining, (pp. 41-50).

Delen, D. (2010). A comparative analysis of machine learning techniques for student retention management. Decision Support Systems, 49(4), 498-506.

Delen, D. (2011). Predicting student attrition with data mining methods. Journal of College Student Retention: Research, Theory and Practice, 13(1), 17-35.

Digication e-Portfolios. (2013, 09 30). Retrieved from http://www.digication.com

Eynon, B. (2009). Making connections: the LaGuardia ePortfolio. Electronic portfolios, 2, 59-68.

Fike, D. S., \& Fike, R. (2008). Predictors of first-year student retention in the community college. Community College Review, 36(2), 68-88.

Flowers, L. A. (2004). Effects of living on campus on African American students' educational gains in college. NASPA Journal, 41(2).

Goodrich, V., Aguiar, E., Ambrose, G. A., Brockman, J., \& Chawla, N. V. (2014). Integration of ePortfolios in a First Year Engineering Course for Measuring Student Engagement. ASEE Annual Conference \& Exposition.

Herzog, S. (2006). Estimating student retention and degree-completion time: Decision trees and neural networks vis-`a-vis regression. New Directions for Institutional Research, 2006(131), 17-33.

Johnson, L. F., Levine, A., Smith, R., \& Stone, S. (2010). The Horizon report: 2010 edition.

Knight, W. E., Hakel, M. D., \& Gromko, M. (2008). The Relationship Between Electronic Portfolio Participation and Student Success. Association for Institutional Research.

Kuh, G. D., Hu, S., \& Vesper, N. (2000). "They Shall Be Known By What They Do": An Activities-based Typology of College Students. Journal of College Student Development, 41(2), 228-44. 
(2014) Engagement vs Performance: Using Electronic Portfolios to Predict First Semester Engineering Student Persistence. Journal of Learning Analytics, 1 (3), 7-33.

Lauría, E. J. (2012). Mining academic data to improve college student retention: An open source perspective. The $2^{\text {nd }}$ International Conference on Learning Analytics and Knowledge, Vancouver, Canada, ACM, NY. (pp.139-142).

Li, Q., Swaminathan, H., \& Tang, J. (2009). Development of a classification system for engineering student characteristics affecting college enrollment and retention. Journal of Engineering Education, 98(4), 361-376.

Lin, J. J., Imbrie, P., \& Reid, K. J. (2009). Student Retention Modelling: An Evaluation of Different Methods and their Impact on Prediction Results. Research in Engineering Education Sysmposium, (pp. 1-6).

Lin, S.-H. (2012). Data mining for student retention management. Journal of Computing Sciences in Colleges, 27(4), 92-99.

Lopez-Fernandez, O., \& Rodriguez-Illera, J. L. (2009). Investigating university students' adaptation to a digital learner course portfolio. Computers \& Education, 52(3), 608-616.

Luna, J. (2000). Predicting student retention and academic success at \{New Mexico Tech\}. Master's thesis, New Mexico Institute of Mining and Technology.

MacGregor, J., \& Leigh Smith, B. (2005). Where are learning communities now? National leaders take stock. About Campus, 10(2), 2-8.

Mendez, G., Buskirk, T. D., Lohr, S., \& Haag, S. (2008). Factors associated with persistence in science and engineering majors: An exploratory study using classification trees and random forests. Journal of Engineering Education, 97(1), 57-70.

Nandeshwar, A., Menzies, T., \& Nelson, A. (2011). Learning patterns of university student retention. Expert Systems with Applications, 38(12), 14984-14996.

Pascarella, E. T. (2006). How college affects students: Ten directions for future research. Journal of College Student Development, 47(5), 508-520.

Pascarella, E. T., Terenzini, P. T., \& Blimling, G. S. (1994). The impact of residential life on students. Realizing the educational potential of residence halls, 22-52.

Pittman, K. (2011). Comparison of data mining techniques used to predict student retention. ProQuest.

Price, M. (2006). Purpose, Audience, and Engagement in Spelman College's eFolio Project. Handbook of Research on EPortfolios, 259.

Quinlan, J. (1986). Induction of Decision Trees. Machine Learning, 1(1), 81-106.

Quinlan, J. R. (1993). C4. 5: programs for machine learning (Vol. 1). Morgan kaufmann.

Ring, G., Weaver, B., Jones Jr, J. J., \& others. (2009). Electronic portfolios: engaged students create multimedia-rich artifacts. Journal of the research center for educational technology, 4(2), 103114.

Seidman, A. (2005). College student retention: Formula for student success. Greenwood Publishing Group.

Thammasiri, D., Delen, D., Meesad, P., \& Kasap, N. (2013). A Critical Assessment of Imbalanced Class Distribution Problem: The Case of Predicting Freshmen Student Attrition. Expert Systems with Applications. 
(2014) Engagement vs Performance: Using Electronic Portfolios to Predict First Semester Engineering Student Persistence. Journal of Learning Analytics, 1 (3), 7-33.

Tinto, V. (2012). Leaving college: Rethinking the causes and cures of student attrition. University of Chicago Press.

Veenstra, C. P., Dey, E. L., \& Herrin, G. D. (2009). A model for freshman engineering retention. Advances in Engineering Education, 1(3), 1-31.

Xenos, M., Pierrakeas, C., \& Pintelas, P. (2002). A survey on student dropout rates and dropout causes concerning the students in the Course of Informatics of the Hellenic Open University. Computers \& Education, 39(4), 361-377.

Yadav, S. K., Bharadwaj, B., \& Pal, S. (2012). Data mining applications: A comparative study for predicting student's performance. arXiv preprint arXiv:1202.4815.

Yancey, K. B. (2009). Reflection and electronic portfolios. BL Cambridge, S. Kahn, DP Tompkins, \& KB Yancey Electronic portfolios, 2, 5-16.

Yu, C. H., DiGangi, S. A., Jannasch-Pennell, A., Lo, W., \& Kaprolet, C. (2007). A Data-Mining Approach to Differentiate Predictors of Retention. Online Submission.

Zhang, G., Anderson, T. J., Ohland, M. W., \& Thorndyke, B. R. (2004). Identifying Factors Influencing Engineering Student Graduation: A Longitudinal and Cross-Institutional Study. Journal of Engineering Education, 93(4), 313-320.

Zhang, Y., Oussena, S., Clark, T., \& Hyensook, K. (2010, June). Using data mining to improve student retention in higher education: a case study. International Conerence on Enterprise Information Systems. 